\title{
Preface to the special issue on ubiquitous user modeling and user-adapted interaction
}

\author{
Tsvi Kuflik $^{1}$ • Judy Kay ${ }^{2}$ • Aaron Quigley ${ }^{3}$
}

Today, computer systems are an integral part of almost every service we use and many devices we rely on in daily life. For example, we may interact directly with devices such as ATMs, information kiosks or ticket machines or with online services for movie rental or shopping. Alternatively many services operate in the background, to support activities such as food ordering, medical treatment, flight booking and boarding and many more. Such services have the potential to be personalized to users' preferences and needs in ways that make them more friendly and useful. To achieve this, systems need knowledge of who is using them or possibly "a target user". However, it is impractical to repeatedly ask users for their preferences and needs; that would be time consuming for the user, inefficient and irritating. In addition, services are now delivered everywhere and in changing environments, i.e. the context of use can vary in terms of changing devices, Internet connectivity and location, along with sensed, inferred or implicit inputs (Quigley 2009). In short, we are approaching an era of truly ubiquitous personalization.

\footnotetext{
$凶 \quad$ Tsvi Kuflik

tsvikak@is.haifa.ac.il

Judy Kay

Judy.Kay@sydney.edu.au

Aaron Quigley

aquigley@acm.org

1 Information Systems Department, The University of Haifa, Mount Carmel, 31905 Haifa, Israel

2 Computer Human Adaptive Interaction Research Group (CHAI), Human Centred Technology Cluster (HCT), The University of Sydney, 1 Cleveland Street, NSW 2006 Sydney, Australia

3 SACHI, School of Computer Science, The University of St Andrews, North Haugh, St Andrews, Fife KY16 9SX, UK
} 
This era is supported by ubiquity of personal technology such as smart mobile devices and wearables. Along with this, there are new social trends in how technologies are used and shared. We are approaching a time when, no matter where we go, we will be able to receive services tailored to our personal preferences and context.

This growth and change motivated a 2012 workshop on Ubiquitous User Modeling on the state of the art and current challenges in Ubiquitous User Modeling, held in Haifa, Israel. During three days of intensive workshop activities, we shared insights and discussed diverse topics, including representations and management of ubicomp user models, cross-system and cross-domain personalisation. We also explored privacy, the Internet of Things and links with emerging interfaces based on gaze and situated displays. We discussed particular contexts of ubicomp personalisation, such as museums and activity modelling. The group concluded that the development of user modelling research and ubiquitous computing had proceeded for too long as parallel streams. We noted that the last UMUAI special issue in this area had been in 2005 and a great deal has happened since then. Notably, much of Weiser's vision (Weiser 1991) has been explored in research and widely deployed hardware and systems. The very basis of this vision has been questioned (Aylett and Quigley 2015) and despite progress, many challenges remain. Some of the particularly challenging remaining aspects relate to usability, interfaces and interaction (Quigley 2009) and the management of personal data and empowering people to manage the personalisation that is central to ubicomp (Abowd 2012). The challenges highlighted for personalisation (Abowd 2012; Kuflik et al. 2012) are precisely where user modelling research has a key role to play in ubicomp. This was the motivation for the current special issue.

This special issue has four papers representing core areas in user modelling research.

The first paper, When User Modeling Intersects Software Engineering: the Infobead User Modeling Approach, by Dim, Kuflik and Reinhartz-Berger, builds upon the body of work on user model servers and shells. It tackles the challenge of creating a user modeling ubicomp infrastructure. It provides an elegant approach to storing ubicomp data, based on the core unit, an info-bead, so that this can support reuse of the model by multiple applications and systems. It was used for a sophisticated deployment in an archeological museum, which has been instrumented to provide fine-grained location tracking of visitors. This framework tackles head on the difficulty of creating such stores and the paper reports an evaluation of its use by students who built diverse user models across several domains and then reused each other's user models. The paper also identifies a comprehensive set of evaluation criteria for user modeling frameworks and describes this system, as well as other major ones from the literature.

Statistical Models for Unobtrusively Detecting Abnormal Periods of Inactivity in Older Adults, by Moshtaghi, Zukerman and Russell, explores the detection of anomalous periods of inactivity as indicators of unsafe events, such as falls, in a home. In-home monitoring systems for older people are seen as a canonical example of the use of, and need for, ubicomp systems. This paper employs two statistical distributions to model normal periods of inactivity: Pareto and hyperexponential. Two methods were developed to estimate the latter: Direct Estimation (DE) and Coefficient of Variation $(\mathrm{CV})$. The estimated distributions were used to build models of abnormally long periods of inactivity, which were evaluated using a function that balances false alerts 
against detection delay. The evaluation on two real-life datasets shows that CV and DE outperform a state-of-the-art baseline.

Trust-based Decision-Making for Smart and Adaptive Environments by Hammer, Wißner and Andre, asks the important question: "when proactive decisions are undertaken by a system, how can we ensure they match users preferences to maintain trust in the system?" A system which fails to achieve this match can result in negative sentiments, to start, and eventually, abandonment. The authors present the User Trust Model based on Bayesian Networks that supports the automatic choice of adequate system reactions. The model incorporates aspects of comfort, transparency, controllability, privacy, reliability, security, credibility and seriousness. The paper explores how such models can be constructed, drawing upon data from an online survey. A smart office setting is explored for integration along with the results of a live study and a user experience survey. By rating the trustworthiness of individual actions, the participants explored and validated the utility of the proposed model as they sought to relinquish control to a proactive decision making system. The presented results can be used to further inform the development of such trust models and can be extended into new settings.

Augmenting Service Recommender Systems by Incorporating Contextual Opinions from User Reviews by Chen and Chen explores the recommendation process with context-independent preferences and aspect-level context-dependent preferences from user-generated reviews. These two sources are combined with a linear-regressionbased algorithm and tested with two real-life service datasets of hotel and restaurant reviews. The testing is compared with three prior approaches (Context Freer, Context Pre-filter and Simple Connecter) and reports on improved recommendation accuracy. Accuracy here is based on Hit Ratio at top-N recommendations and Mean Reciprocal Rank. The approach relies on three steps, contextual opinion extraction, contextindependent preference inference and context-dependent preference inference. These feed into the recommendation algorithm based on linear regression. Based on the user's current context, recommendation results are delivered in a ranking step.

We would like to acknowledge the help of the reviewers who provided detailed and helpful reviews, so enabling authors to improve the papers that were finally accepted to the special issue. Finally, we acknowledge the role of UMUAI editor in chief in managing the reviewing of papers authored by the guest editors.

\section{References}

Abowd, G.D.: What next, ubicomp?: celebrating an intellectual disappearing act. In: Proceedings of the (2012) ACM Conference on Ubiquitous Computing (pp. 31-40). ACM. (2012)

Aylett, M., Quigley, A.: The Broken Dream of Pervasive Sentient Ambient Calm Invisible Ubiquitous Computing in Proceedings of the 33rd Annual ACM Conference Extended Abstracts on Human Factors in Computing Systems - CHI EA '15, 425-435 (2015)

Kuflik, T., Kay, J. Kummerfeld, Kummerfeld, B.: Challenges and Solutions of Ubiquitous User Modeling. In: Kuflik, Kruger, (eds.) Ubiquitous Display Environments, pp. 7-31. Springer-Verlag, (2012)

Quigley, A.: From GUI to UUI: Interfaces for Ubiquitous Computing in Ubiquitous Computing Fundamentals, Edited by John Krumm, Chapman and Hall/CRC 2009, pp 237-284 (2009)

Weiser, M.: The computer for the 21st century. Scientific American 265(3), 94-104 (1991) 